Ann. Zootech., I970, 19 (I), 53-66.

\title{
ÉVOLUTION DU GAIN DE POIDS VIF \\ ET DE L'ÉTAT D'ENGRAISSEMENT DES VACHES TARIES AU PATURAGE
}

\author{
C. BÉRANGER, M. NEGRIN et C. MALTERRE \\ avec la collaboration technique de Marie-Thérèse Gozzelino, G. Cuylle et G. Villeneuvi: \\ Station de Recherches sur l'Élevage des Ruminants, \\ Centre de Recherches de Clermont-Ferrand, 63 -Saint-Genès-Champanelle \\ Institut national de la Recherche agronomique
}

\section{RÉSUMÉ}

Nous avons décrit la cinétique de reprise de poids vif, ainsi que la reconstitution des tissus musculaire et adipeux des vaches laitières destinées à la boucherie, engraissées au pâturage après tarissement.

A cet effet, nous avons comparé d'une part sur 24 vaches deux durées d'engraissement de 46 et 80 jours, d'autre part sur 45 vaches trois durées d'engraissement, respectivement de 28,55 et 84 jours.

Les vaches aduites amaigries sont capables, après tarissement, d'avoir au pâturage un gain de poids vif journalier très élevé, comparable à celui des mâles en croissance de même race, mais au cours des 6-7 premières semaines d'engraissement seulement, un très net ralentissement de la croissance ayant lieu au-delà de cette période.

Il apparaît que la composition de la carcasse varie très peu avec la durée de la période d'en. graissement entre 30 et 90 jours, car l'adipogénèse semble aller de pair avec la protéinogénèse.

\section{INTRODUCTION}

La viande dite de bouf est fournie en France pour 45 p. Ioo par des bovins abattus en cours de croissance (bœufs, taureaux, génisses) et pour 55 p. Ioo par des vaches adultes, abattues en fin de carrière après avoir produit du lait et des veaux.

Les animaux en croissance développent d'abord leur tissu nerveux, leur squelette, leurs muscles, puis leurs dépôts adipeux (selon le schéma classique de Hammond) et sont abattus, selon le type de production, à un poids et à un âge variables déterminés par un état d'engraissement optimum. 
Les vaches adultes utilisent de façon plus ou moins intensive leurs réserves adipeuses et, éventuellement, leurs masses musculaires pour couvrir les besoins énergétiques et azotés afférant à la production laitière. Avant d'être abattues, elles doivent donc reconstituer leur musculature et leurs dépôts adipeux. Toutefois, pour obtenir des carcasses de qualité, il faut permettre aux vaches de développer au maximum leurs muscles sans former une quantité excessive de dépôts adipeux, en d'autres termes favoriser la protéinogénèse et limiter l'adipogénèse ; celle-ci est en effet plus coûteuse sur le plan nutritionnel et entraîne, lorsqu'elle est excessive, une diminution de la valeur commerciale des carcasses.

Les études concernant la croissance musculaire ont généralement porté sur des animaux jeunes, mais le phénomène de la reconstitution des masses musculaires et des réserves adipeuses, ainsi que les variations de composition corporelle chez les vaches adultes, n'ont fait l'objet d'aucun travail systématique; quelques vaches ont été cependant utilisées dans des études d'ensemble sur la composition corporelle des bovins (CALLOW, I947), mais sans que les résultats obtenus aient été reliés au passé des animaux. En Norvège Homs et al. (1965) ont étudié la mobilisation des réserves corporelles en fonction du niveau d'alimentation : d'après leurs résultats, lorsque des vaches laitières sont trop grasses, une diminution du niveau énergétique (de 25 p. Ioo par rapport aux besoins) durant 5 mois, en début de lactation, permettrait de réduire notablement (de I6 à 25 p. Ioo) la teneur en matières grasses des carcasses tout en maintenant la production laitière ; mais c'est la seule étude expérimentale réalisée sur la composition des carcasses de vaches laitières depuis les travaux de Moulton aux U. S. A. en rg2r. En effet, dans la plupart des pays, les carcasses de vaches laitières étant en général destinées à la fabrication de viande hachée, de charcuterie ou de conserves, leur qualité n'a donc pas beaucoup retenu l'attention des chercheurs et des techniciens de l'élevage. En France, au contraire, les vaches représentent environ la moitié de la viande de "bœuf " consommée ; en đépit de cela leur préparation pour la boucherie est très variable et très empirique.

Nous avons entrepris depuis I 963 une série d'études portant sur la reconstitution des tissus musculaires et adipeux chez la vache adulte. En l'absence de connaissances antérieures, nous avons commencé par décrire l'évolution du gain de poiđs et de la composition corporelle au cours de l'engraissement, avant d'étudier l'influence des facteurs alimentaires et physiologiques. Nous avons d'abord engraissé au pâturage des vaches adultes taries, amaigries ou même émaciées, telles qu'elles se présentent dans la zone Normande. Nous rapportons ici les résultats de trois expériences réalisées au cours des printemps de I963, I964 et I967, au domaine expérimental de 1'I. N. R. A. au Pin-au-Haras (Orne).

\section{MA'TÉRIEI, E'T MÉTHODES}

Pour décrire l'évolution du gain de poids vif et de sa composition, nous avons constitué des lots de vaches comparables que nous avons abattus au bout de 6-7 et IO-I 2 semaines dans la première expérience, et au bout de 4,8 et I 2 semaines dans la seconde. N'ayant pu, au cours de ces deux premières expériences mesurer les quantités d'herbe consommées, nous avons dans la troisième, distribué de l'herbe coupée à un lot de vaches comparable aux précédents et abattu au bout de ro semaines (tabl. I). 
$\mathrm{Au}$ cours des trois expériences nous avons utilisé des vaches de race $N o r m a n d e$, taries, maigres et non gestantes. Nous avons constitué des blocs de 2 ou 3 animaux en fonction de leur âge apprécié par l'état de la dentition, de leur poids et de leur état d'engraissement apprécié subjectivement par le toucher des dépôts adipeux sous-cutanée les plus caractéristiques.

$9 \mathrm{p}$. Ioo des animaux ont été éliminés en raison de maladie ou de vêlage en cours d'expérience. Cela explique, en particulier pour la deuxième expérience, les écarts entre les poids moyens initiaux des différents lots (tabl. 2).

\section{TABLEAU I}

\section{Caractéristiques des expériences}

\begin{tabular}{|c|c|c|c|}
\hline & $1^{\text {re }}$ expérience & 2 e expérience & $3^{e}$ expérience \\
\hline Année $\ldots \ldots \ldots \ldots \ldots \ldots \ldots$ & 1963 & $196^{\prime} \mathbf{x}$ & 1967 \\
\hline $\begin{array}{l}\text { Nombre d'animaux mis en } \\
\text { expérience ............. } \\
\text { Nombre de lots expérimentaux. } \\
\text { Nombre d'animaux éliminés . } \\
\text { Date de mise à l'herbe ...... }\end{array}$ & $\begin{array}{c}28 \\
2 \\
4 \\
11 \text { mai }\end{array}$ & $\begin{array}{c}48 \\
3 \\
3 \\
27 \text { avril }\end{array}$ & $\begin{array}{c}10 \\
1 \\
1 \\
26 \text { avril }\end{array}$ \\
\hline
\end{tabular}

\section{Alimentation et conditions de vie}

Entre leur achat et le début de l'expérience, les animaux sont restés à l'extérieur dans des parcs et ont reçu du foin et de la paille, puis ils ont été mis à l'herbe et l'expérience a débuté 8 à Io jours plus tard.

Au cours des deux premières expériences, les animaux ont tous été conduits dans les mêmcs conditions au pâturage en rotation sur plusieurs parcelles de prairies naturelles bien fertilisées (8o N-8o P-8o K) et assez productives, dont la flore était constituée essentiellement de houlque laineuse, de pâturin, d'agrostis et de trèfle blanc ; ils ont exploité les deux premiers cycles de croissance de l'herbe et ont toujours disposé à volonté d'une herbe jeune et de bonne qualité.

Dans la troisième expérience, les animaux, après trois semaines de pâturage, ont été engraissés à l'auge en stabulation libre durant toute la période expérimentale et ont reçu à volonté de l'herbe

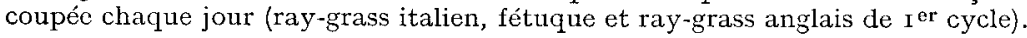

\section{Mesures}

\section{Gain de poids vif.}

Les animaux ont été pesés au début (8-Io jours après la mise à 1'herbe) et à la fin de chaque période expérimentale ainsi que toutes les deux semaines, dans les conditions suivantes : une première pesée a été faite le matin vers 9-Io heures après le premier cycle de pâturage et une seconde l'après-midi vers 15-16 heures, les animaux étant maintenus à jeun depuis la pesée du matin. Ce sont les moyennes de ces deux pesées qui ont été utilisées pour calculer les gains de poids des animaux et établir leur courbe de gain de poids vif (fig. I).

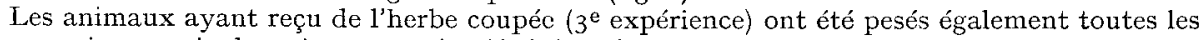
deux semaines, mais deux jours consécutifs à la même heure.

\section{Mensurations corporelles.}

Pour caractériser le format des animaux et leur développement corporel, nous avons mesuré au début et à la fin de l'expérience la hauteur au garrot, le tour de poitrine et la largeur aux trochanters.

L'état d'engraissement a été apprécié subjectivement toutes les semaines et avant le départ à l'abattoir, et noté de o à 6 (o pour un animal très émacié, 6 correspondant à un animal trop gras pour la demande actuelle). L'appréciation se faisait par le toucher des dépôts adipeux sous-cutanés 
dont on estime l'épaisseur au niveau de l'attache de la queue (" abords »), du repli de la peau qui, de la cuisse, rejoint la paroi de l'abdomen ("grasset ") et de l'arrière de l'épaule (" paleron ").

\section{Abattage.}

Les vaches d'un même lot n'ont pu être abattues simultanément mais au cours d'une période de 3 semaines, les animaux les plus gras ayant été abattus les premiers sans tenir compte de l'appariement initial. Toutefois, tous les animaux de la troisième expérience ont été abattus simultanément.

Les animaux ont été transportés à l'abattoir du C. N. R. Z. distant de $200 \mathrm{~km}$, un ou deux jours avant l'abattage pour la première et la troisième expérience et le jour même à un abattoir beaucoup plus proche ( $5 \mathrm{~km})$ pour la seconde expérience.

\section{Étude de la carcasse et de la composition chimique de la viande}

A l'abattage, on a mesuré les rendements en carcasse et le poids des éléments du "cinquième quartier "(différence entre le corps vide et la carcasse). La carcasse de chaque animal a été notée au point de vue de sa conformation et de son état d'engraissement et a fait l'objet d'un certain nombre de mensurations et de pesées après découpe : mesure de la longueur de la carcasse et de la cuisse (distance jarret-symphyse), épaisseur de la cuisse et du faux-filet (VISSAC, I959), poids du "pan traité " (Dumont, 1956), ainsi que de l'ensemble épaule + collier, pour les animaux de la première expérience.

Pour caractériser la proportion respective đu muscle et du tissu adipeux, à défaut de pouvoir disséquer totalement la carcasse, on a pesé les dépôts adipeux de la cavité générale (dépôt adipeux péritonéal, mésentérique,...), le dépôt adipeux périrénal ainsi que précrural. La " II ${ }^{\mathbf{e}}$ côte " (GEAY, BERANGER, 1969), prélevée sur le "pan traité " de la demi-carcasse gauche de chaque animal a été disséquée ; on a pesé séparément l'os, les muscles et les dépôts adipeux ; on a broyé et échantillonné la viande désossée dont on a déterminé la teneur en matière sèche et matières grasses (extraction à l'éther de pétrole après hydrolyse acide).

\section{Quantités d'herbe consommées.}

On a mesuré les quantités d'herbe verte et de matière sèche ingérées tous les jours pour le lot de vaches de la $3^{\mathrm{e}}$ expérience, ainsi que la digestibilité de cette herbe sur un lot de 4 béliers en cage à bilan. La valeur énergétique moyenne de l'herbe a été déterminée chaque semaine à partir du coefficient de digestibilité de la matière organique, selon la formule proposée par BREIREM (1954) et utilisée par Demargutlly (1965).

\section{RÉSULTATS}

\section{Évolution du poids et de l'état d'engraissement (Première et deuxième expériences)}

Dans la première expérience, les durées moyennes d'engraissement ont été de 46 et de 80 jours respectivement pour les lots I et II. Dans la seconde, elles ont été respectivement pour les lots I, II et III de 28,55 et 84 jours.

\section{Gain de poids vif.}

Les résultats obtenus au cours des deux expériences concordent (tab1. 2, fig. I) : au cours des 6-7 premières semaines le gain de poids vif est toujours très élevé (supérieur à I ooo g/jour), puis il diminue très rapidement jusqu'à des valeurs de 400-500 g/jour. Dans la première expérience les vaches ont gagné $62 \mathrm{~kg}$ de poids vif durant les 46 premiers jours, puis seulement $2 \mathrm{I} \mathrm{kg}$ durant les 34 jours suivants ; dans la deuxième expérience, elles ont gagné respectivement, pour les 42 et 55 premiers jours, 50 et $57 \mathrm{~kg}$, puis seulement $7 \mathrm{~kg}$ pour les 29 jours supplémentaires. 
Les variations individuelles de reprise de poids sont considérables (le coefficient de variation se situe entre 32 et 56 p. Ioo selon les lots) ; sur ces 69 animaux, nous n'avons pu mettre en évidence aucune liaison entre le croît et l'âge ou l'état d'engraissement initial des animaux, en raison du nombre limité d'individus.

TABLEAU 2

Gain de poids des vaches dans les deux premières expériences

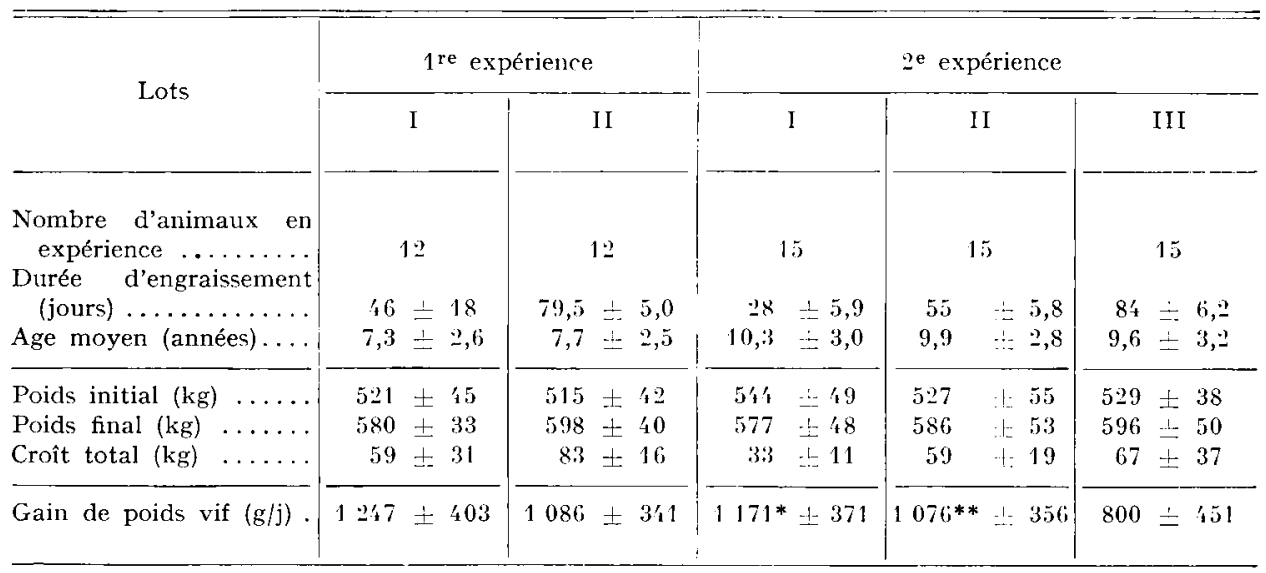

* Différence significative à $\mathbf{P}<0,05$ avec le lot $1 \mathrm{II}$.

** Différence hautement significative à $\mathrm{P}<0,01$ avec le lot Il I (calculs effectués par la méthode des couples).

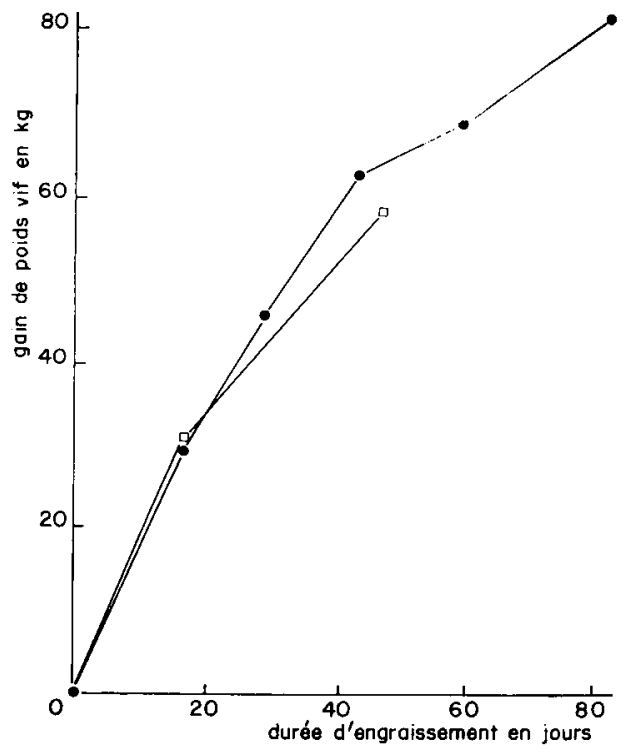

liw. I. - Evolution du gain de poids vif en fonction du temps (Première expérience) 


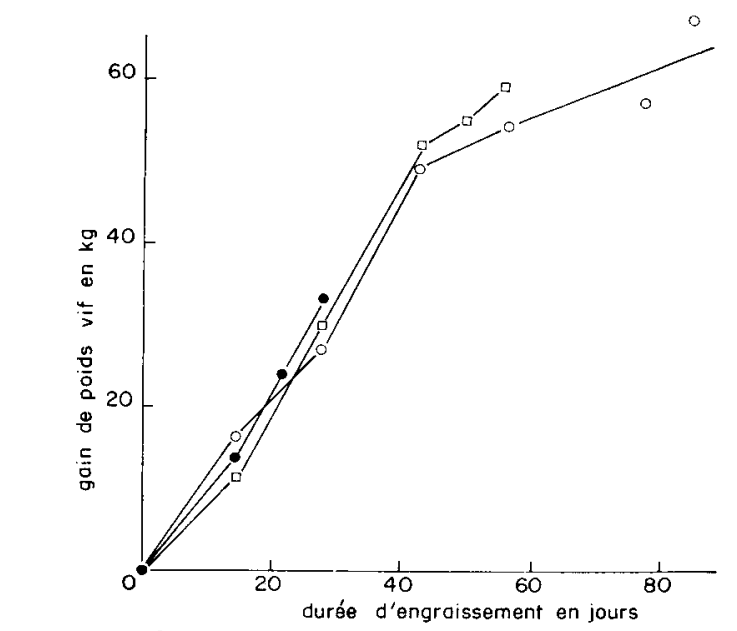

FIG. I. - Evolution du gain de poids vif en fonction du temps

(Deuxième expérience)

Rendement et conformation de la carcasse.

Dans chacune des deux expériences, les rendements en carcasse, exprimés en pourcentage $d u$ poids vif ou du poids vif vide, n'ont pas varié de façon significative avec la durée d'engraissement.

La compacité de la carcasse et l'épaisseur des muscles au niveau du fau $\mathrm{x}$-filet (région à développement tardif) ont légèrement augmenté au cours de l'engraissement dans la seconde expérience; en revanche, les autres rapports utilisés pour mesurer la conformation des carcasses, de même que les proportions des différentes parties, n'ont pas varié de façon notable (tabl. 3).

État d'engraissement.

Les divers critères utilisés pour mesurer 1'état d'engraissement des carcasses n'ont pas permis de mettre en évidence de différences significatives en fonction de la durée d'engraissement (tab1. 4). En particulier, la composition de la " I I ${ }^{\mathrm{e}}$ côte $)$ ne varie pas de façon significative; l'analyse chimique des muscles et dépôts adipeux de la " II e côte ", effectuée sur tous les animaux de la seconde expérience, n'a montré aucune différence significative dans les teneturs en eat, matières grasses et matières minérales (tab1. 5). Cependant, on observe dans la seconde expérience une légère augmentation de la proportion de dépôts adipeux et de matières grasses dans la " II ${ }^{\mathrm{e}}$ côte " en fonction de la durée d'engraissement; cette évolution apparaît plus nettement au niveau du tissu adipeux périrénal, sans toutefois être significative.

Dans tous les cas, la proportion de dépôts adipeux dans la carcasse n'était pas excessive, mais satisfaisante pour la boucherie ( $20 \mathrm{p}$. Ioo environ dans la " II ${ }^{\mathbf{e}}$ côte ", ce qui correspondrait chez un animal jeune à I7 p Ioo de dépôts adipeux dans la carcasse, (GEAY et BÉRANGER, I969).

Sur l'ensemble des individus, trop peu nombreux, aucune relation n'a pu être mise en évidence entre l'état d'engraissement et le poids de carcasse d'une part, le croît et 1'âge d'autre part. 
ENGRAISSEMENT DE LA VACHE ADUL'TE

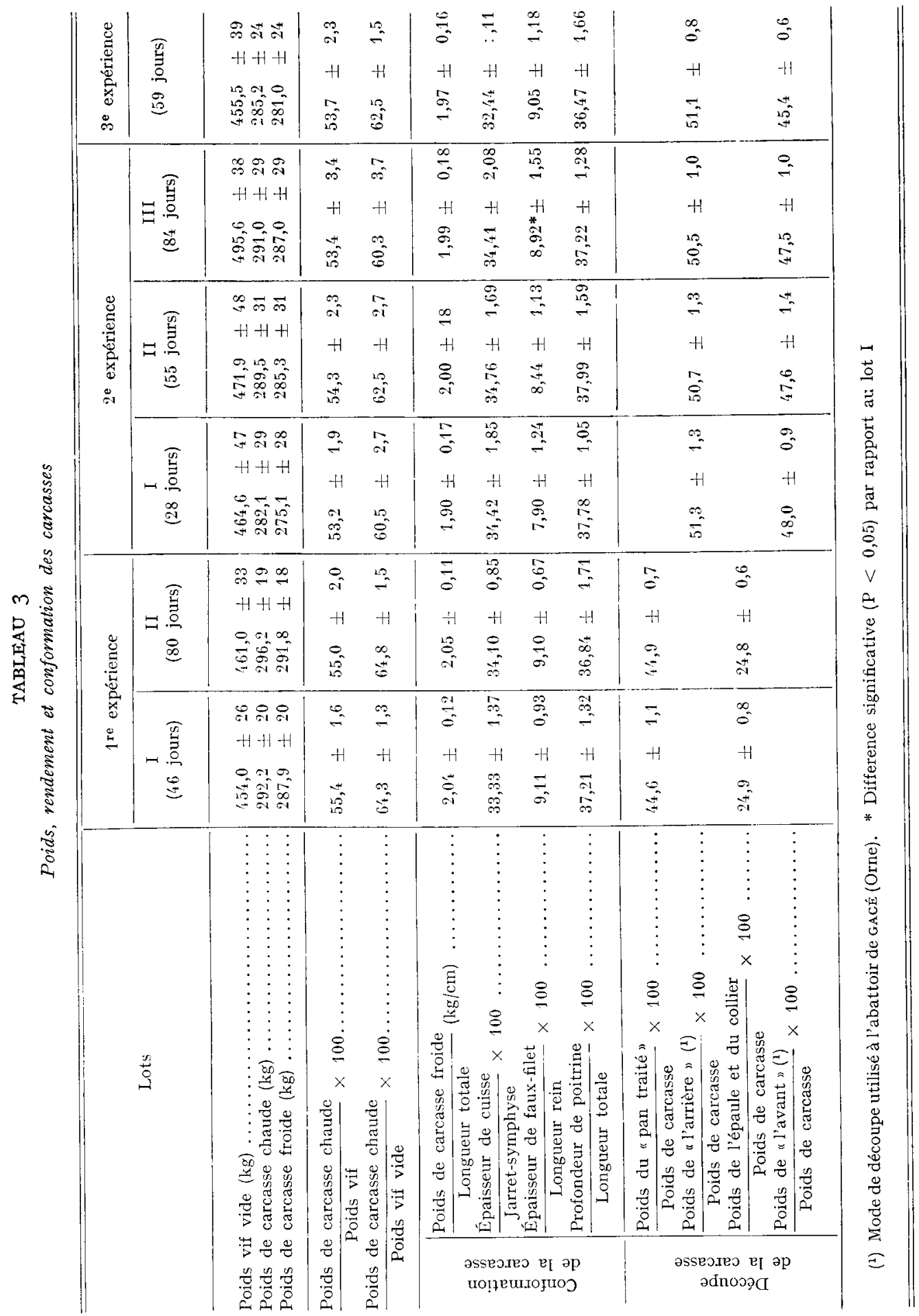




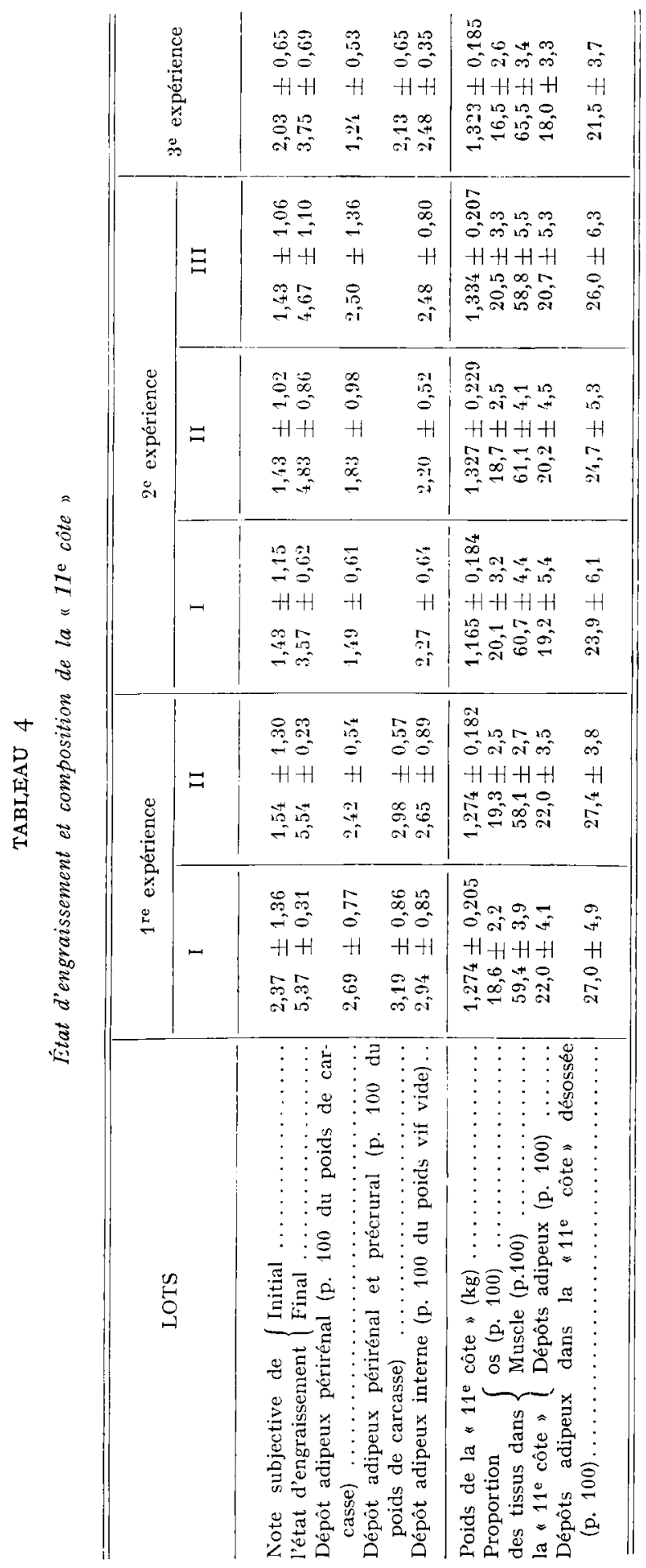


On peut donc conclure que la valeur "bouchère " des animaux (rendement en carcasse, conformation, état d'engraissement) n'a pas varié de façon sensible entre le $30^{\mathbf{e}}$ et 1 e $90^{\mathbf{e}}$ jour d'engraissement.

\section{TABLEAU 5}

Composition chimique de la viande désossée de la $11^{\mathrm{e}}$ côte

(Deuxième expérience)

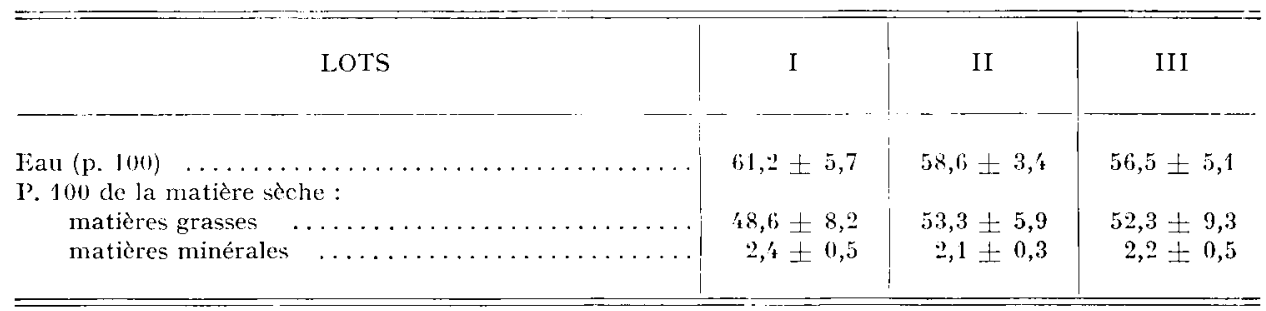

\section{Quantités d'herbe ingérées (troisième expérience)}

Au cours de la $3^{\mathrm{e}}$ expérience, les animaux recevant de l'herbe coupée durant toute la période d'engraissement (59 jours) ont réalisé des gains de poids vif comparables et même légèrement supérieurs à ceux obtenus au pâturage dans les autres expériences et ont produit des carcasses de poids et d'état d'engraissement voisins (cf. tabl. 3, 4 et 6) ; ils ont donc vraisemblablement consommé des quantités d'herbe et d'énergie équivalentes à celles ingérées par les animaux au pâturage.

TABLEAU 6

Gain de poids et quantités ingérées

(Troisième expérience)

\begin{tabular}{|c|c|}
\hline 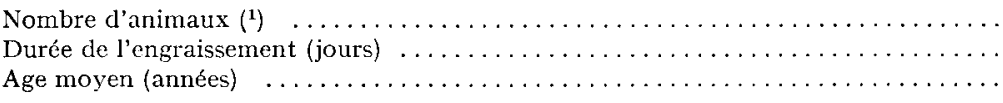 & $\begin{array}{l}8 \\
59 \\
5,9 \pm 1,2\end{array}$ \\
\hline 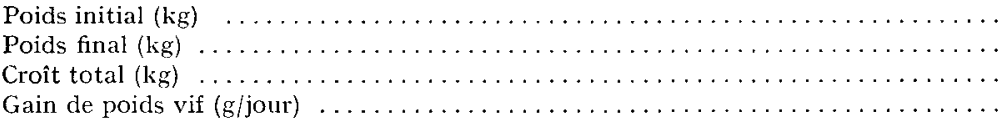 & $\begin{array}{r}494,4 \pm 30,7 \\
576,0 \pm 36,7 \\
81,6 \pm 15,8 \\
1384 \pm 268\end{array}$ \\
\hline 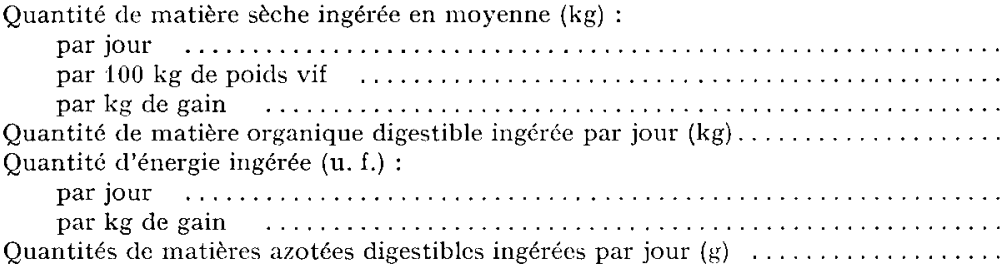 & $\begin{array}{r}12,92 \\
2,39 \\
9,34 \\
7,86 \\
8,1^{\prime} \\
6,10 \\
655\end{array}$ \\
\hline
\end{tabular}

(1) 10 animaux durant les trois premières semaines, 9 ensuite, dont un a été saisi à l'abattoir par suite d'entérite. 
La quantité de matière sèche ingérée par les animaux fut assez élevée durant l'ensemble de la période expérimentale $(2,39 \mathrm{~kg}$ de matière sèche par Ioo $\mathrm{kg}$ de poids vif, tabl. 6) : elle a augmenté au cours des trois premières semaines puis a diminué et s'est stabilisé à la fin de l'engraissement (fig. 2). La quantité de matière organique digestible ingérée par jour est restée à peu près constante, tandis que celle des matières azotées digestibles ingérées a diminué de I ooo à $400 \mathrm{~g}$ par jour.
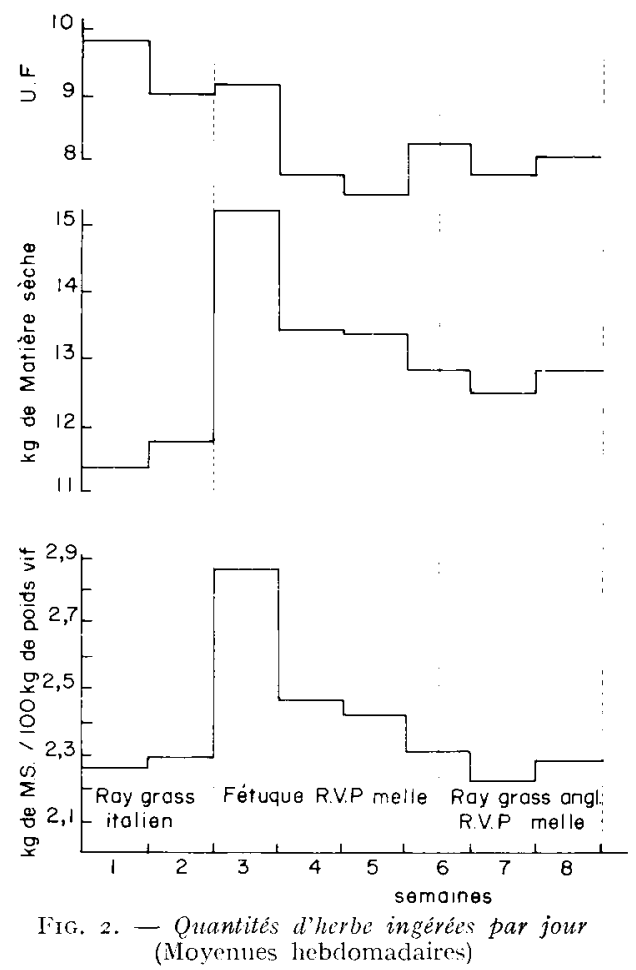

I) urant les 59 jours d'engraissement, les vaches ont ingéré en moyenne $8,4 \mathrm{I}$ U.F. (soit 6,I U.F./ $\mathrm{kg}$ de gain); elles ne disposaient donc que de 4,2 U.F./jour en plus des besoins d'entretien pour réaliser un gain de poids vif qui a été de I $384 \mathrm{~g} /$ jour ; leur besoin énergétique de production serait done de 3-3, I U. F. par kg de gain.

Selon la méthode de calcul proposée par 1'Agricultural Research Council (I965), la quantité d'énergie métabolisable ingérée a été en moyenne de 26,89 Mcal par jour (valeur corrigée). Si les besoins d'entretien de ces vaches, pesant $535 \mathrm{~kg}$ en moyenne, étaient de II,I7 Mcal, elles auraient disposé de I5.72 Mcal pour l'accroissement de leur masse corporelle. Si l'énergie métabolisable pour l'engraissement était utilisée avec un rendement de 44,6 p. Ioo (A. R. C. p. 200, tabl. 6-4), les vaches auraient pu fixer 7 ooo kcal par jour. Iin admettant une valeur calorifique du croît de 4500 à 5 OOO kcal $/ \mathrm{kg}$ (BIAXTER et WAINMAN pour les bovins de plus de 2 ans, KELLNER pour les bœufs âgés), cette ingestion aurait permis un gain de I 400 à I 500 g/jour qui est égal ou légèrement supérieur à celui observé. On peut donc penser que la valeur calorifique du croît de ces vaches serait légèrement supérieure à 5 ooo kcal et leur besoin de production voisin de 3 U.F. par $\mathrm{kg}$ de gain. 


\section{DISCUSSION ET CONCLUSIONS}

\section{Aspects méthodologiques}

La reprise de poids vif et l'engraissement des vaches de réforme sont complexes car ils résultent à la fois des conditions actuelles d'exploitation et d'alimentation de la vache et des conditions passées. Les différences individuelles sont par suite beaucoup plus importantes que chez les animaux jeunes dont le passé est généralement moins variable. La constitution de couples ou de blocs d'animaux comparables a été très difficile, car nous ne disposions pas de vaches dont le passé fût suffisamment connu (âge exact, nombre de vêlages, niveau de production laitière, alimentation et conduite antérieures) ; l'appréciation de l'état d'engraissement au début de la période expérimentale est trè̀ subjective ; l'élimination de certains animaux en cours d'expérience (gestation, maladie) a détruit en partie les lots constitués au clépart. Ėnfin, les animaux d'un même lot n'ont pu être abattus exactement à la date prévue ; en effet, pour des raisons pratiques, il a fallu échelonner les abattages afin de pouvoir effectuer les mesures sur les carcasses de tous les animaux. Pour réaliser ces études, il faut utiliser des effectifs importants en provenance de nombreux élevages et par suite subir des risques, sanitaires notables.

Iìn outre, la mesure de la reprise le poids par pesée est moins précise que dans le cas des jeunes bovins à l'engrais, car les variations de poids du contenu digestif semblent être plus grandes et la durée de la périorle expérimentale plus courte ; pour atténuer ces effets, il faut faire des pesées nombreuses et fréquentes, ce qui est difficile à réaliser au pâturage.

D'autre part, aucun des critèrej utilisés pour mesurer la proportion de dépôts adipeux dans la carcasse n'est pleinement satisfaisant; les résultats obtenus sur des jeunes bovins permettent de penser que la composition de la " I I ${ }^{\mathrm{e}}$ côte ", séparée du " pan traité ", reflète assez bien celle de la carcasse $\langle r=0,948$ entre le pourcentage de dépôts adipeux dans la côte et dans la carcasse des taurillons étudiés par GrAY et BÉraNGEr, I969), mais il faudrait savoir dans quelle mesure cette méthode peut s'appliquer aux vaches adultes. Il aurait été souhaitable de pouvoir abattre des vaches maigres au début de la période expérimentale, pour connaitre réellement la composition du croît.

Enfin, les vaches de la troisième expérience ont consommé, à l'auge, une herbe différente (composition botanique et mode d'exploitation) de celle qui a été pâturée dans les autres expériences.

\section{Aspects physiologiques et nutritionnels}

Les deux études descriptives de l'engraissement des vaches ont donné des résultats très concordants. L'évolution du gain de poids vif chez les vaches taries est très différente de celle des bœufs en croissance âgés de 2 ou 3 ans, de même race et de même format $\left({ }^{1}\right)$; il est très important durant les $6-8$ premières semaines d'engrais-

(1) Par exemple les boufs normands engraissés au pâturage dans le mêmo donaine expérimental. 
sement (I IOO-I $300 \mathrm{~g} / \mathrm{jour}$ ) presqu'aussi élevé que celui des bœufs, mais par suite il diminue beaucoup plus tôt et beaucoup plus rapidement; la vitesse de croissance

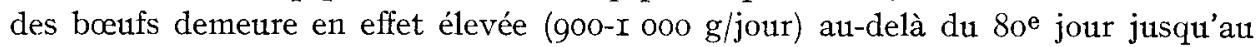
${ } 5^{\circ}{ }^{\mathrm{e}}$ jour d'engraissement. I a phase intensive de reprise de poids s'arrête rapidement chez les vaches parce qu'elles sont adultes (4 à I4 ans) et que leur croissance est terminée ; elles ont un certain format auquel correspond un poids maximum qui ne saurait être dépassé sauf par un engraissement excessif. La reprise de poids durant les 2-3 mois d'engraissement doit dépendre de l'écart entre le poids initial de la vache et ce poids maximum lié à son format; il doit aussi dépendre de 1'âge de l'animal ; malheureusement le nombre insuffisant d'individus utilisés dans cette étude ne permet pas de mettre ces phénomènes en évidence.

Les trois expériences réalisées montrent que le développement des dépôts adipeux est beaucoup plus rapide chez les vaches que chez les bœufs en croissance. En effet, des mesures effectuées dans le cadre d'une autre étude (BÉRANGER, MALTERRE) sur les carcasses de 26 vaches maigres abattues au début de la lactation (en moyenne au cours $\mathrm{du} 4^{\mathrm{e}}$ mois, époque où les réserves adipeuses n'ont pas encore été reconstituées) montrent que la " II ${ }^{\mathrm{e}}$ côte " contenait alors en moyenne Io p. Ioo de dépôts adipeux. On peut penser que les vaches taries, très amaigries, que nous avons utilisées n'étaient certainement pas plus grasses ; or, au bout de 5-6 semaines la " II ${ }^{\mathrm{e}}$ côte " contient déjà 20 p. Ioo de dépôts adipeux, puis cette proportion semble augmenter très lentement et faiblement jusqu'à la ${ }^{2}{ }^{\text {e }}$ semaine. Au contraire, chez le bœuf l'état d'engraissement augmente régulièrement avec le temps et de plus en plus rapidement au cours des derniers mois, lorsque la vitesse de croissance diminue; il faut en moyenne $5_{50}$ jours d'engraissement pour que la " II ${ }^{\mathbf{e}}$ côte " de bœufs normands âgés de $2-3$ ans contienne $22 \mathrm{p}$. Ioo de dépôts adipeux.

Les vaches adultes reconstituent donc simultanément leur musculature et leurs réserves adipeuses et atteignent très rapidement un état d'engraissement optimum pour la boucherie, avant même d'avoir réalisé leur reprise de poids maximum.

Le croît considérable des vaches comportant une proportion élevée de graisse correspond à une quantité d'énergie fixée très importante. Cela résulte à la fois d'un niveau d'ingestion élevé et d'une bonne utilisation de l'énergie. En effet, les mesures effectuées dans la $3^{\mathrm{e}}$ expérience révèlent un appétit relativement élevé pour des vaches taries (JourneT) qui peut être lié à l'absence de fotus et de dépôts adipeux, et résulte aussi sans doute de la valeur nutritive élevée de l'herbe de printemps. D'autre part, les calculs effectués à partir de cəs quantités ingérées (cf. p. 6I et 62) semblent indiquer que 1'efficacité de la transformation énergétique en croit est du même ordre que chez les autres catégories de bovins à l'engrais (la valeur de $\mathrm{K} f$, rendement de l'utilisation métabolisable, paraît correcte, de même que des besoins de 3 U.F. par kg de gain).

L'importance relative de l'adipogénèse au cours de cette phase de reprise rapide de poids pourrait s'interpréter de la façon suivante : les vaches ingèrent alors une quantité importante d'éléments nutritifs (matière organique et matières azotées digestibles) ; leur potentiel de protéinogénèse étant sans doute limité parce qu'elles sont adultes, une part importante de l'énergie ingérée est fixée sous forme de graisse. Ensuite, en raison de la diminution de l'appétit et de la valeur nutritive de 1'herbe, la quantité d'énergie ingérée diminue (fig. 2) ; elle continue à assurer la protéinogénèse, mais la lipogénèse est moins importante qu'au début et la composition corpo- 
relle évolue peu au cours du second et du troisième mois d'engraissement. Dans cette hypothèse, il devrait être possible de modifier la composition des carcasses, soit en limitant le niveau énergétique de la ration au cours des premières semaines, soit en favorisant la protéinogénèse aux dépens de la lipogénèse en modifiant la nature des produits terminaux de la digestion ou l'équilibre hormonal de l'animal.

\section{Aspects techniques}

Quelle que soit leur durée d'engraissement, les animaux utilisés dans ces expériences ont été jugés corrects pour la boucherie ; même au bout de 4 semaines d'engraissement leur "état " paraissait suffisant. La proportion de dépôts adipeux dans la " $I^{\mathrm{e}}$ côte " n'a pas été supérieure à 26 p. Ioo, à l'exception de quelques vaches déjà grasses au début de l'expérience. Par conséquent, c'est la diminution du gain de poids journalier au-dessous d'un certain seuil économique, plus que l'état d'engraissement apparent, qui doit déterminer le stade optimum d'abattage. En moyenne, au bout de $50-60$ jours le gain de poids journalier devient faible, son cô̂t devient très élevé sans que cela soit compensé par une amélioration notable de la qualité des carcasses. En revanche, si l'on abat les animaux avant 40 jours d'engraissement, la qualité de leur carcasse est certes correcte, mais on diminue leur poids et on n'utilise pas tout le potentiel de récupération de l'animal.

Les vaches laitières adultes, taries et amaigries, sont donc capables de reprendre rapidement, en $50-70$ jours, 60 à $80 \mathrm{~kg}$ de poids vif soit 30 à $40 \mathrm{~kg}$ de carcasse et de produire ainsi des carcasses de qualité convenable. Les risques d'engraissement excessif semblent minimes au cours des go premiers jours, ce qui doit permettre d'adapter dans une certaine mesure la vente aux fluctuations des prix entre le $40^{\mathrm{e}}$ et le goe jour d'engraissement.

Durant environ deux mois, la vache tarie engraissée au pâturage se révèle un bon transformateur permettant une bonne valorisation des excédents d'herbe de printemps ; dans ces conditions, le coût de son engraissement doit être faible, et elle cesse d'exploiter les prairies lorsque la production d'herbe diminue. Beaucoup de vaches taries en fin d'hiver doivent ainsi pouvoir produire économiquement des carcasses de bonne qualité.

Ces résultats obtenus avec des vaches de race Normande devraient être vérifiés sur d'autres races. En effet, il existe certainement des différences raciales, mais ce paramètre n'a jamais fait l'objet d'études systématiques.

Soixante jours environ paraissent être la durée optimale d'engraissement au-delà de laquelle la diminution du gain de poids quotidien entraîne un coût énergétique trop important, sans que cela soit compensé par une amélioration de la qualité des carcasses obtenues.

Resu pour publication en décembre 1969.

\section{REMERCIEMEN'TS}

Cette étude a été réalisée grâce à l'aide de la D. G. R. S. 'T. (contrat: 63 FR 288) à qui nous exprimons nos remerciements.

Nous tenons à remercier également M. le Professeur Charlet qui nous a prodigué ses consejls et son aide stimulante tout au long de cette étude. 


\section{SUMMARY}

\section{PROGRESSION OF IIVE WEIGHT GAIN AND FATTENING \\ OF COWS DRIED OFF AT PASTURE}

We have studied the progression of live weight gain and carcass composition of dairy cows to be slaughtered which were fattened at pasture after drying off.

Lots of comparable cows were slaughtered after 46 and 80 days of fattening in a first experiment ( 24 cows), and after 28,55 and 84 days of fattening in a second experiment ( 45 cows) In a third experiment, a lot of 9 cows, comparable to the preceeding ones, was fattened for 7 o days with cut grass, and the quantities consumed were measured (table I).

After drying off, lean adult cows may have a very large daily live weight gain, which is comparable to that of growing males of the same breed, but which lasts only the first 6-7 weeks of fattening. After that, weight gain is very little (table 2).

Carcass composition seemed to vary very little with the length of fattening (between 30 and 90 days), where as it should progress rapidly during the first month (tables 3,4 and 5 ).

Dried off lean cows have a large appetite which allows them to ingest 26 to $27 \mathrm{Mcal} /$ day of metabolizable energy in the form of grass. They thus have a weight gain higher than I $\mathrm{kg}$ per day, with good feed efficiency, during the first $5^{\circ}$ days of fattening (table 6).

About two months seems to be the optimum length of fattening time. After that, a decrease of daily weight gain involves a too great feed cost which is not compensated for by the carcass quality obtained.

\section{RÉFÉRENCES BIBLIOGRAPHIQUES}

Agricultural Research Council, 1965 . The nutrient requirements of farm livestock. $\mathrm{n}^{0} 2$ Ruminants, London. Béranger C., Malterre C. (Résultats non publiés).

Dumont B. L., I956. La découpe du pan traité. F. E. Z. Commission bovine. Madrid.

Bretrem K., I954. Die Nettoenergie als grundlage der bewertung der futtermittel, in : Nehring K. Ioo jahre Möckern. Die bewertung der futterstoffe und andere probleme der Tiernahrung. Berlin, Deutsche Akad. der Lanndwirtschaftswissenschaften, t. II, 97-Io8.

Callow E. H., 1947. Comparative studies of meat. J. Agric. Sci., 37, 113-132.

Geay Y., BÉranger C., rg69. Estimation de la composition de la carcasse de jeunes bovins à partir de la composition d'un morceau monocostal au niveau de la II e côte. Ann. Zootech., 18, 65-77.

Homb T., Matre T., Opstvedt J., Arvid L., Breirem K., r965. Underfeeding of fat dairy cows the last months before slaughtering. Inst. Anim. Nutr. A gricultural college of Norway. Report $n^{\circ}{ }_{127 .}$

JoURnet M. (communication personnelle).

Moulton C. R., Trowbridge P. F., Haigh L. D., r923. Studies in animal production. V. Changes in the composition of the Nature Dairy Cow, while fattening. University of Missouri. Agric. exp. Stat. Res. Bull. 61.

Vissac B., 1959. Rapport sur des recherches françaises intéressant le testage des taureaux sur les aptitudes à la production de viande de leurs descendants. C. R. des journées d'études de la F. E. Z. n $n^{\circ} 700 /$ 59 , Belgrade. 